Canadian Journal of Higher Education

Revue canadienne d'enseignement supérieur

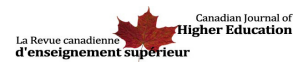

\title{
Le rêve inachevé d'étudier et de socialiser en français en toute sécurité linguistique : les perspectives d'étudiants franco-ontariens
}

\section{Johanne Jean-Pierre}

Volume 47, numéro 3, 2017

URI : https://id.erudit.org/iderudit/1043241ar

DOI : https://doi.org/10.7202/1043241ar

Aller au sommaire du numéro

\section{Éditeur(s)}

Canadian Society for the Study of Higher Education

ISSN

2293-6602 (numérique)

Découvrir la revue

Citer cet article

Jean-Pierre, J. (2017). Le rêve inachevé d'étudier et de socialiser en français en toute sécurité linguistique : les perspectives d'étudiants franco-ontariens. Canadian Journal of Higher Education / Revue canadienne d'enseignement supérieur, 47(3), 120-135. https://doi.org/10.7202/1043241ar

\section{Résumé de l'article}

Suivant une démarche interprétative, cet article examine les perspectives d'étudiants franco-ontariens rapportées lors d'une étude qualitative exploratoire. Des entretiens semi-structurés séquentiels menés en profondeur ont été effectués à Ottawa et à Toronto entre janvier et juin 2014 auprès de 18 étudiants franco-ontariens. Les résultats indiquent que les participants franco-ontariens qui fréquentent des établissements postsecondaires à vocation bilingue souhaitent à la fois une amélioration de l'accès aux études en français et de la qualité de la langue d'instruction ainsi que de meilleures possibilités de socialiser en français. Les participants qui fréquentent des établissements à vocation française ont affirmé être satisfaits de leur expérience d'apprentissage, tandis que ceux inscrits dans des établissements à vocation anglaise ont déploré un sentiment d'isolement linguistique. De plus, le manque d'occasions pour socialiser en français et inclure des réalités franco-ontariennes dans les curriculums, ainsi que les incidents d'intimidation linguistique alimentent l'insécurité linguistique.
Copyright (c) Johanne Jean-Pierre, 2017

Attribution-NonCommercial-No Derivative Works 2.5 Canada
Ce document est protégé par la loi sur le droit d'auteur. L'utilisation des services d'Érudit (y compris la reproduction) est assujettie à sa politique d'utilisation que vous pouvez consulter en ligne.

https://apropos.erudit.org/fr/usagers/politique-dutilisation/ 


\title{
Le rêve inachevé d'étudier et de socialiser en français en toute sécurité linguistique : les perspectives d'étudiants franco-ontariens
}

Johanne Jean-Pierre
Université Ryerson

Résumé

Suivant une démarche interprétative, cet article examine les perspectives d'étudiants franco-ontariens rapportées lors d'une étude qualitative exploratoire. Des entretiens semi-structurés séquentiels menés en profondeur ont été effectués à Ottawa et à Toronto entre janvier et juin 2014 auprès de 18 étudiants franco-ontariens. Les résultats indiquent que les participants franco-ontariens qui fréquentent des établissements postsecondaires à vocation bilingue souhaitent à la fois une amélioration de l'accès aux études en français et de la qualité de la langue d'instruction ainsi que de meilleures possibilités de socialiser en français. Les participants qui fréquentent des établissements à vocation française ont affirmé être satisfaits de leur expérience d'apprentissage, tandis que ceux inscrits dans des établissements à vocation anglaise ont déploré un sentiment d'isolement linguistique. De plus, le manque d'occasions pour socialiser en français et inclure des réalités franco-ontariennes dans les curriculums, ainsi que les incidents d'intimidation linguistique alimentent l'insécurité linguistique.

\begin{abstract}
Using an interpretative approach, this paper examines the perspectives of Franco-Ontarian postsecondary students employing an exploratory qualitative research. Between January and June 2014, in-depth semi-structured interviews were conducted in Ottawa and Toronto with 18 Franco-Ontarian postsecondary students. The findings reveal that participants who study in bilingual postsecondary institutions would like to have access to courses in French, as well as see improvement in the quality of the language of instruc-
\end{abstract}


tion, and the opportunities to socialize in French. Participants who study in French-language postsecondary institutions express satisfaction with their learning experience, whereas participants who study in English-language postsecondary institutions express sentiments of linguistic isolation. In addition, the lack of socialization opportunities in French and the underrepresentation of Franco-Ontarian realities in the curriculum combined with instances of linguistic intimidation fuel linguistic insecurity.

\section{Introduction}

C'est en Ontario que l'on retrouve le plus grand nombre de francophones hors Québec; en effet, le recensement de 2006 dénombrait plus de 500 ooo Franco-Ontariens (Office des affaires francophones, 2009). Tandis que plusieurs études canadiennes examinent les expériences au niveau postsecondaire d'immigrants (Kilbride \& D'Arcangelo, 2002), d'autochtones (Hampton \& Roy, 2002; Pidgeon, Archibald, \& Hawkey, 2014), de parents qui concilient leurs responsabilités parentales et les études (Van Rhijn, Quosai, \& Lero, 2011), ainsi que d'étudiants ayant un handicap (Dwyer, 2000), celles des minorités de langue officielle sont moins souvent étudiées (Labrie \& Lamoureux, 2016). Cet article analyse les préoccupations soulevées par des étudiants de communautés francophones ontariennes en situation minoritaire dans une étude exploratoire qualitative. Pour ce faire, il s'appuie sur une démarche interprétative et sur la théorie symbolique interactionniste, en plus d'aborder des thèmes tels que l'apprentissage et la socialisation en français, l'identité et la sécurité linguistiques. Le but de cet article est de mieux saisir la dimension microsociologique du vécu quotidien d'étudiants franco-ontariens dans des établissements postsecondaires à vocations française et anglaise.

\section{Le contexte}

Le gouvernement de l'Ontario a reconnu que, tout comme les étudiants autochtones, ceux qui ont un handicap et ceux de première génération, la population étudiante francophone est sous-représentée au niveau postsecondaire (Rae, 2005). Les jeunes FrancoOntariens (20,3\%) et les Acadiens du Nouveau-Brunswick (19\%) sont moins nombreux à obtenir un diplôme postsecondaire que ne le sont les francophones d'autres provinces et de territoires hors Québec (28,4 \%) (Pilote \& Magnan, 2014). Plusieurs auteurs ont d'ailleurs analysé cet enjeu de la sous-représentation des Franco-Ontariens aux études postsecondaires (Frenette \& Quazi, 1996; Labrie, Lamoureux, \& Wilson, 2009). Afin de pallier les besoins en éducation des communautés franco-ontariennes, les gouvernements fédéral et ontarien ont conclu L'entente Canada-Ontario relative à l'enseignement dans la langue de la minorité et à l'enseignement de la langue seconde officielle 2013-2014 à 2017-2018 (Gouvernement de l'Ontario, 2014). De plus, la Politique d'aménagement linguistique de l'Ontario (Ministère de l'Enseignement supérieur et de la Formation professionnelle, 2011) accorde une attention toute particulière à la qualité de l'instruction, à l'accès aux programmes en français et au rendement des différents établissements offrant une formation en français.

Bien que les efforts des membres de la communauté, des établissements postsecondaires et des gouvernements soient de bon augure, certaines améliorations semblent 
nécessaires pour pouvoir étudier en français en toute sécurité linguistique en Ontario. En effet, à la suite de nombreuses plaintes de francophones liées au manque de programmes en français dans le centre et le sud-ouest de l'Ontario, le Commissariat aux services en français a publié un rapport comportant plusieurs recommandations en 2012. Ce dernier souligne la nécessité d'améliorer l'accès aux études postsecondaires en français et recommande l'ouverture d'une université de langue française dans le centre sud-ouest de la province. Tout comme Frenette et Quazi (1996), le rapport conclut que le processus d'analyse décisionnelle d'une telle initiative ne devrait pas se limiter à un simple calcul de l'offre et de la demande, mais plutôt envisager le recrutement d'étudiants internationaux et d'immersion française.

À première vue, la nécessité d'offrir plus d'occasions d'apprentissage en français au niveau postsecondaire en Ontario peut surprendre. En effet, un large éventail de collèges et d'universités offrent des programmes en français, comme l'illustre le Tableau 1. En examinant de plus près la vocation linguistique de ces établissements, on s'aperçoit cependant que la majorité d'entre eux sont à vocation bilingue et que la possibilité de suivre l'ensemble des cours en français dans un programme donné ou encore de socialiser en français au quotidien varie d'un campus à l'autre. La Cité collégiale, le Collège Boréal et l'Université de Hearst (affiliée à l'Université Laurentienne) se révèlent être les seuls établissements à offrir une éducation et un environnement exclusivement en français.

Tableau 1. Aperçu des établissements postsecondaires offrant des programmes en français en Ontario

\begin{tabular}{|c|c|c|c|}
\hline & Nom de l'établissement & Fondation & Vocation \\
\hline \multirow[t]{3}{*}{ Collèges } & Collège d'Alfred-Université Guelph & 1981 & Bilingue \\
\hline & La Cité collégiale & 1989 & Française \\
\hline & Collège Boréal & 1995 & Française \\
\hline \multirow[t]{9}{*}{ Universités } & Université d’Ottawa & 1848 & Bilingue \\
\hline & Université Saint-Paul ${ }^{1}$ & 1848 & Bilingue \\
\hline & Collège militaire royal du Canada & 1852 & Bilingue \\
\hline & Collège universitaire dominicain & 1900 & Bilingue \\
\hline & Université de Hearst ${ }^{2}$ & 1953 & Française \\
\hline & Collège Glendon-Université York & 1959 & Bilingue \\
\hline & Université Laurentienne & 1960 & Bilingue \\
\hline & Université de Sudbury² & 1960 & Bilingue \\
\hline & $\begin{array}{l}\text { Institut d'études pédagogiques de l'Ontario-Université } \\
\text { de Toronto }\end{array}$ & 1979 & Bilingue \\
\hline
\end{tabular}

${ }^{1}$ Affiliée à l'Université d'Ottawa; ${ }^{2}$ Affiliées à l'Université Laurentienne

L'accès à une éducation postsecondaire en français est important pour la participation des Franco-Ontariens à ce niveau d'études, mais également pour le soutien de la vitalité linguistique, que l'on peut définir comme tout ce qui fait en sorte qu'un groupe puisse fonctionner de façon active et participative en tant qu'entité distincte. Selon Giles, 
Bourhis, \& Taylor (1977), la vitalité linguistique est influencée par trois facteurs : le statut de la langue, la démographie et les institutions. À l'exception du Nouveau-Brunswick, toutes les communautés de langue française hors Québec font face à une vitalité linguistique en déclin en raison d'un faible taux de natalité, de l'exogamie et d'un faible taux de transmission intergénérationnelle de la langue (Castonguay, 2002; Landry, 2014).

Or, la présence d'un ensemble d’institutions peut renforcer la présence du fait français. Cette complétude institutionnelle, que Breton (1964) définit comme la capacité d'un groupe à obtenir des services dans plusieurs secteurs d'activités (religieuses, politiques, professionnelles et récréatives) à travers un réseau d'institutions, favorise le maintien, la reproduction et la transmission d'un patrimoine culturel. À l'instar des écoles de langue française qui améliorent la transmission et le maintien de la langue française (Churchill, 2016), les établissements postsecondaires ontariens peuvent jouer un rôle collectif déterminant dans le cadre des formations professionnelles, collégiales ou universitaires qu'ils offrent.

La socialisation dans les milieux familial et scolaire est fondamentale pour la transmission de la langue et la survie du français en milieu minoritaire (Pilote, Magnan, \& Vieux-Fort, 2010), et celle du niveau postsecondaire occupe une place particulièrement importante, puisque les études collégiales et universitaires façonnent la transition entre l'école secondaire et une carrière professionnelle. Comme seulement $43 \%$ des FrancoOntariens entreprennent des études postsecondaires uniquement en français (Pilote \& Magnan, 2014), il semble pertinent d'explorer l'interprétation du vécu postsecondaire d'étudiants inscrits dans des collèges et universités avec différentes vocations linguistiques.

\section{Cadre théorique}

L'analyse des résultats qui suit a été effectuée selon la théorie symbolique interactionniste. Dès 1934, le sociologue George Herbert Mead suggère que la capacité de penser et de prendre des décisions, ainsi que la construction identitaire, sont influencées par la communauté à laquelle appartient un individu. Chaque communauté adhère à un ensemble de caractéristiques et de symboles dont le sens est partagé par l'ensemble de ses membres. Autrement dit, nul ne se développe en vase clos. Pour comprendre les choix effectués par tout un chacun ou par des groupes sociaux, on se doit d'analyser non seulement leurs interactions sociales, mais aussi l'interprétation de ces interactions, sans quoi il demeure impossible de saisir un phénomène social dans son ensemble ou même les conséquences qui en découlent (Blumer, 1969). En effet, comme le montrent les études contemporaines en sociologie de la culture, la façon dont on agit dans différents contextes est déterminée par le sens que l'on associe à une action ou à un symbole (Alexander, 2013; Anderson, 1999; Hall \& Lamont, 2013). De surcroît, l'approche symbolique interactionniste permet d'explorer les réactions des personnes et des groupes en situation d'inégalité et d'exclusion sociale, qui peuvent parfois diverger de celles du reste de la population (Anderson \& Snow, 2001).

Plusieurs études qualitatives révèlent que l'identité linguistique est une construction sociale en constante évolution et qu'elle est accompagnée d'une pluralité de discours et de références (Dallaire, 2008; Pilote \& Joncas, 2016; Pilote \& Magnan, 2012). Cette identité est également fluide, en mouvance et façonnée par les pratiques sociales (Gérin-Lajoie, 2011, 2014). Ainsi, on constate par exemple qu'un nombre croissant de membres des minorités de langue officielle s’identifient comme bilingues en raison de leurs interactions 
quotidiennes, qui requièrent l'usage à la fois de l'anglais et du français (Dallaire \& Denis, 2005; Gérin-Lajoie, 2011; Landry, Deveau, \& Allard, 2008).

Au-delà de la question de l'identité linguistique se pose celle de l'insécurité linguistique. La langue française au Canada est parlée de façon très variée sur les plans lexical, phonétique et morphologique (Boudreau, 2014; Boudreau \& Dubois, 2001; Chevalier, 2008; Lozon, 2002). Il arrive néanmoins que des francophones provenant d'un milieu majoritaire (France, Québec) ne reconnaissent pas la légitimité du français parlé par ceux issus de milieux minoritaires (LeBlanc, 2010). La façon dont une personne parle français peut dès lors provoquer, par le biais d'une intervention, d'un commentaire ou d'un geste menaçant ou humiliant, des incidents d'intimidation linguistique (Fédération de la jeunesse francoontarienne, 2014). On parle également de linguicisme, c'est-à-dire de discrimination basée sur la langue parlée (Bourhis, Sioufi, \& Sachdev, 2012). Dans cet article, l'insécurité linguistique réfère avant tout à la perception personnelle d'avoir de faibles capacités langagières en français, particulièrement en comparaison avec les francophones qui proviennent de milieux linguistiques majoritaires (Larouche \& Hinch, 2012). À cela s'oppose la sécurité linguistique, soit la perception d'être capable de parler sa langue sans gêne et sans malaise dans différents contextes (Lozon, 2002). L'insécurité linguistique mine les efforts déployés pour le maintien et la transmission de la langue française dans les milieux où le français est restreint presque uniquement à la sphère privée.

Dans le cadre des expériences d'études postsecondaires, il est tout indiqué d'examiner l'interprétation que les étudiants font de leur apprentissage et de leurs interactions sociales avec le personnel administratif, leurs collègues de classe et leurs professeurs et chargés de cours. Les extraits narratifs ci-dessous sont tirés des réponses à la dernière question posée lors de l'entretien : "Comment croyez-vous que votre institution postsecondaire a influencévotre sentiment identitaire linguistique? »Cette question permet d'explorer deux questions de recherche : Selon les perceptions des participants, quelles caractéristiques du milieu postsecondaire fréquenté renforcent l'identité linguistique? et Quelles sont celles qui renforcent le sentiment d'insécurité linguistique?

\section{Cadre méthodologique}

Cet article s'appuie sur une partie des résultats d'une étude comparative qualitative exploratoire $^{1}$ portant sur les expériences postsecondaires des minorités de langue officielle ontarienne et québécoise. Parmi les thèmes explorés dans cette étude, on trouve l'identité linguistique, les processus culturels, le bilinguisme et les expériences en milieu postsecondaire. Puisque les participants franco-ontariens ont abordé l'accès aux cours et la socialisation en français, et puisque les participants anglo-québécois n'ont pas exprimé de telles préoccupations, seules les données relatives aux perceptions des étudiants postsecondaires franco-ontariens sont rapportées ici.

Avec une démarche interprétative (Weber, 1949), l'approche symbolique interactionniste a permis d'établir les thèmes émergents des entretiens effectués auprès des participants. Dans le cadre de la collecte des données, j’ai effectué, entre janvier et juin 2014, une série d'entretiens semi-structurés séquentiels menés en profondeur et d'une durée variant de 40 à 90 minutes. La technique des entretiens séquentiels consiste à traiter chaque entretien comme une étude de cas, tout en tentant de reproduire cet entretien avec les participants subséquents (Small, 2009). Les entretiens avec les 
étudiants franco-ontariens ont eu lieu à Ottawa et à Toronto, dans les locaux des campus ou à proximité. La région d'Ottawa, communément appelée la région de Champlain, a été sélectionnée comme lieu stratégique pour recruter des participants en raison du grand nombre de Franco-Ontariens qui y résident (Office des affaires francophones, 2012).

Un total de 18 participants franco-ontariens, avec un nombre égal d'hommes et de femmes, ont été recrutés. Il s'agissait d'étudiants collégiaux ou universitaires, à temps plein ou diplômés au cours des 12 mois précédents. Le recrutement s'est effectué grâce à un effet boule de neige chez les membres d'associations étudiantes et civiles. Le tableau ci-dessous (Tableau 2) résume les caractéristiques démographiques des participants : le sexe, la ou les langues parlées dans la famille immédiate et la vocation linguistique de l'établissement postsecondaire fréquenté. La majorité des participants sont issus de familles endogames, c'est-à-dire de familles où les deux parents parlent français à la maison (on appelle exogames les familles où les parents ne parlent pas la même langue). Parmi les répondants, quatre étaient étudiants au niveau collégial et la moitiéétaient inscrits dans un programme d'études universitaires de premier cycle à l'Université d'Ottawa - cet établissement à vocation bilingue constitue le premier choix des étudiants désirant obtenir un diplôme universitaire en français dans les régions du nord-est, du centre sud-ouest et de l'est de l'Ontario (Labrie \& Lamoureux, 2016). Le fait qu'une grande proportion de participants de notre échantillon étudie à l'Université d'Ottawa facilite donc l'analyse du vécu postsecondaire dans une université très fréquentée par la communauté franco-ontarienne.

Tous les entretiens ont été enregistrés, transcrits et codifiés en plusieurs étapes. Des noms fictifs ont été attribués à chaque participant pour maintenir l'anonymat et la confidentialité. Le logiciel $M A X Q D A$ a été utilisé pour codifier les entretiens par étapes. Une codification a d'abord été effectuée afin de créer des catégories pour l'ensemble des sujets abordés dont celui des études postsecondaires. Ce sujet a ensuite lui-même été divisé en sous-catégories en fonction de thèmes prédominants. Le protocole de recherche a été approuvé par le McMaster Research Ethics Board.

Tableau 2. Aperçu des caractéristiques démographiques des participants francoontariens

\begin{tabular}{ll}
\hline Caractéristiques démographiques & Participants \\
\hline Sexe & Femmes $\mathrm{N}=9$ \\
& Hommes $\mathrm{N}=9$ \\
Environnement familial linguistique & Endogame $\mathrm{N}=11$ \\
& Exogame $\mathrm{N}=7$ \\
Niveau d'études & Collégial $\mathrm{N}=4$ \\
& Universitaire $\mathrm{N}=14$ \\
Vocation linguistique de l'établissement & Unilingue anglaise $\mathrm{N}=4$ \\
postsecondaire fréquenté & Unilingue française $\mathrm{N}=5$ \\
& Bilingue $\mathrm{N}=9$ \\
\hline
\end{tabular}




\section{Analyse des données}

À l'exception d'une seule d'entre eux, tous les participants ont émis des propos concernant certains aspects linguistiques de leur parcours postsecondaire. Leurs préoccupations peuvent être regroupées en deux grands thèmes, analysés séparément ci-dessous :

1. La difficulté d'accéder à des cours en français et la qualité de la langue d'instruction;

2. Le manque d'occasions de socialiser en français, l'insécurité linguistique et les répercussions de ces deux réalités sur l’identité linguistique.

\section{Difficulté d'accéder à des cours en français et qualité de la langue d'instruction}

À l'Université d'Ottawa, université à vocation bilingue, certains programmes sont offerts intégralement en français, certains offrent la majorité des cours en anglais ou en français, et d'autres requièrent que l'étudiant suive la moitié des cours en anglais et l'autre moitié en français. Cela fait en sorte qu'en fonction du programme choisi, l'accès à des cours en français peut varier. Or, un enjeu soulevé par huit des neuf étudiants de cet établissement est précisément un accès parfois difficile aux cours en français dans leurs programmes respectifs. Cet enjeu est de taille, puisque plusieurs d'entre eux ont affirmé que des cours ne sont offerts en français qu'une fois tous les deux ans, ce qui oblige certains étudiants qui tiennent à suivre un cours en français à repousser la fin de leurs études.

Agnès est une étudiante dont les deux parents parlent français à la maison et dont le parcours scolaire s'est déroulé exclusivement dans le système de langue française. Elle a entrepris des études à la Faculté des sciences sociales universitaires de l'Université d'Ottawa avant de transférer à La Cité collégiale pour suivre un programme artistique. Dans l'extrait qui suit, elle raconte comment, dans le premier établissement, elle a dû suivre des cours en anglais pour cheminer dans son programme parce que les cours en question n'étaient pas offerts en français. Agnès pose un regard critique sur cette situation en soulevant le fait que, contrairement à elle, les membres de la majorité linguistique en Ontario n'ont pas à faire ce type de choix.

Ben, par exemple, étant francophone minoritaire en Ontario, mon bac, je n'ai pas pu le terminer en français, par exemple. Il a fallu que je prenne des cours en anglais parce qu'il n'y a pas d'universités unilingues francophones. [...] Je n'ai pas pu terminer toutes mes études dans ma langue tandis que pour la majorité, c'est un acquis.

Même lorsqu'un cours est offert en français pendant une session, le nombre de places disponibles peut se révéler insuffisant pour le nombre d'étudiants francophones et francophiles qui souhaitent le suivre. Judith est étudiante à la Faculté des arts de l'Université d'Ottawa et, comme Agnès, elle a grandi dans une famille avec deux parents parlant français à la maison. Elle a effectuél'ensemble de sa scolarité primaire et secondaire en français et évoque la difficulté de suivre certains cours dans cette langue : " Fait que, la disponibilité des cours n'est pas la même. Même si le cours est offert, t'es pas garantie d'avoir une place dans le cours en français. »

À l'Université d'Ottawa, certains cours sont bilingues et se veulent une occasion d'apprentissage dans les deux langues officielles. Selon Robert, cependant, ces cours 
se déroulent surtout, sinon complètement, en anglais. Il explique comment plusieurs étudiants qui y sont inscrits n'ont qu'une connaissance limitée du français et que, par défaut, l'enseignement et les échanges se font en anglais. Robert a effectué toute sa scolarité en français et, lors de l'entretien, il avait récemment obtenu un baccalauréat, après cinq années d'études en sciences sociales à l'Université d'Ottawa. Même s'il est bilingue et vient d'une famille exogame, il se dit attaché à son identité franco-ontarienne et au fait français en Ontario, et déplore qu'au cours de ses études universitaires, les cours bilingues n'aient pas été véritablement bilingues : « Je me suis inscrit à des cours bilingues. J'ai plusieurs amis qui se sont inscrits à des cours qui étaient supposés être donnés en français et en anglais, et c'est devenu des cours anglophones sans exception. »

Sept des neuf participants ayant fréquenté l'Université d'Ottawa ont rapporté avoir éprouvé de la difficulté à comprendre certains professeurs et chargés de cours, ce qui a rendu leur apprentissage plus ardu. Louis, par exemple, vient d'une famille endogame; il a suivi toute sa scolarité en français et terminait sa deuxième année à l'Université d'Ottawa au moment de l'entretien. Il considère que le fort accent de certains professeurs peut affecter la compréhension du contenu du cours : « Fait qu'ils ont un accent assez prononcé, certains. Il y en a qui n'en ont pas. C'est difficile pis t'utilises la plupart de ton énergie à juste comprendre les mots. Ça affecte un peu la compréhension de la matière. »

Pour Raoul, étudiant à la Faculté des sciences sociales de l'Université d'Ottawa, cette difficulté qu'ont certains professeurs et chargés de cours à s'exprimer en français va même jusqu'à gêner son apprentissage : " On dit qu'on a des professeurs qui parlent français, mais qui ont clairement de la difficulté à parler en français. Oui, ça nuit certainement à mon apprentissage. Si on a de la misère à communiquer entre le professeur et l'étudiant. »

Adélaïde, étudiante dans un programme interdisciplinaire en français à l'Université d'Ottawa, vient d'une famille exogame et a effectué l'ensemble de sa scolarité en français. Elle estime que certains professeurs et chargés de cours qui n'ont pas le français ou l'anglais comme langue maternelle peuvent être difficiles à comprendre.

Je vais avoir, admettons, un enseignant ou un prof qui n'est pas d'ici ou il parle une autre langue qui n'est pas l'anglais ou le français comme première langue, fait qu'on a un petit peu de misère à comprendre quand il parle le français. Comme je ne veux pas dire qu'ils ne sont pas bons à s'exprimer en français, mais on a beaucoup de misère à comprendre lorsqu'ils parlent.

Cinq participants fréquentaient un établissement à vocation de langue française au moment des entrevues. Contrairement aux participants ayant fréquenté un établissement à vocation bilingue, ceux ayant fréquenté un établissement postsecondaire à vocation linguistique française n'ont pas mentionné l'accès aux cours ou la qualité de la langue comme étant un souci lors des entretiens.

\section{Manque d'occasions de socialiser en français, insécurité linguistique et ré- percussions sur l'identité linguistique}

Dans une université à vocation française, la possibilité de socialiser dans cette langue facilite la rencontre d'individus issus de la francophonie canadienne et internationale. Béatrice suggère que cet espace renforce dès lors l'appartenance à la francophonie tout en contribuant à une plus grande solidarité entre francophones. 
Il y a beaucoup de Québécois, de Français qui sont ici. On vient de toutes sortes de différentes réalités. En France, ben le français est majoritaire. Mon amie québécoise ne connaissait vraiment rien des minorités francophones en Ontario pis maintenant, elle m'aide avec le RÉFO [Regroupement étudiant franco-ontarien]. Elle aide vraiment à monter une fierté francophone en Ontario. Pis moi, ben, c'est ça, là. On est le trio Québec, France et Franco-Ontarienne.

L'apport institutionnel des collèges et des universités à des expériences de socialisation significatives est important sur les plans individuel et collectif parce qu'il renforce l'attachement linguistique (Pilote, Magnan, \& Vieux-Fort, 2010). Les participants euxmêmes rapportent que leurs expériences de socialisation et d'apprentissage ont eu un effet sur leur identité linguistique. La moitié des répondants a évoqué que le fait de fréquenter leur établissement postsecondaire avait eu des répercussions sur leur identité linguistique. Agnès, qui, après avoir étudié à l'Université d'Ottawa, a transféré à La Cité collégiale, considère que cette dernière a davantage consolidé son identité linguistique francophone : "Je dirais que ça l'a renforcée depuis que j'étudie en français à 100 \% et je travaille en français à $100 \%$. Je ne parle presque plus anglais. »

Parmi les répondants pour qui l'établissement n'a pas eu d'importance, l'établissement à vocation linguistique française représente surtout une continuité entre l'expérience scolaire antérieure en français et la formation professionnelle. C'est le cas pour Blanche, une étudiante à La Cité collégiale provenant d'une famille endogame :

C'est un peu compliqué à dire parce que tu sais, à [ville d'origine], je parlais juste en français déjà d'avance dans mon école pis dans la ville. Des fois, un peu en anglais, mais ici, c'est la même chose. Je vais parler en français à l'école, des fois en dehors de l'école comme en ville, je vais parler en anglais.

Tout comme Blanche, Emma, étudiante à l'Université Saint-Paul, considère que son établissement n'a eu aucun effet sur son identité linguistique et précise que son quotidien se passe surtout en français : "Non, il n'y a eu aucun effet. Je ne crois pas que... Je travaille, j'étudie en français. » La possibilité de fonctionner en français pourrait donc faire en sorte que l'on ne considère pas que l'établissement postsecondaire influence l'identité linguistique.

Si les étudiants inscrits dans des établissements postsecondaires de langue française se disent satisfaits de leur socialisation en français, seuls quatre des treize participants fréquentant un établissement bilingue ou de langue anglaise sont de cet avis, alors que les neuf autres estiment manquer d'occasions de socialiser dans leur langue.

Dans les établissements postsecondaires à vocation bilingue comme l'Université d'Ottawa, les activités organisées par les associations étudiantes et l'administration de l'université sont souvent annoncées comme ayant lieu dans les deux langues officielles. Charles, originaire d'une famille exogame et étudiant de premier cycle dans un programme bilingue, est l'un de ceux pour qui l'université a su consolider l'identité linguistique francophone bilingue : "Ça a beaucoup renforcé. J'ai trouvé qu'ils mettent beaucoup d'efforts dans le bilinguisme ».

Cependant, Marguerite, qui terminait ses études de premier cycle à la Faculté des sciences sociales à la même université au moment de l'entretien, a un point de vue 
différent. Outre le Carrefour francophone (salon étudiant en français sur le campus) et certaines activités spécifiques en français, cette étudiante ayant effectué l'ensemble de ses études primaires et secondaires dans cette langue affirme que, malgré ses efforts pour établir une vie universitaire en français, son identité linguistique est mise à mal sur le campus. Elle raconte :

Donc là, ça fait juste réaffirmer le fait que je ne pense pas qu'on est sécuritaire dans sa langue sur le campus. Des espaces francophones existent ici au Carrefour francophone, mais c'est rare. L'espace francophone existe pendant une joute d'impro. Il va exister pendant un club de débats. Pis je pense que c'est la seule activité où est-ce qu'on peut se sentir à l'aise d'être francophone, puis de s'exprimer dans la langue de notre choix pis de se faire comprendre. Sinon, malgré les efforts de la Fédération étudiante - et je le sais là, ces gens-là, ils font de grands efforts pour que ce soient des activités bilingues. En réalité, je pense que ça ne se reflète pas, malheureusement.

Sans surprise, l'isolement linguistique, c'est-à-dire ce que l'on ressent lorsqu'on ne peut pas parler sa langue avec une autre personne dans un contexte donné, est encore plus important pour les quatre étudiants franco-ontariens qui poursuivent des études dans un établissement postsecondaire de langue anglaise. Jean, par exemple, a effectué toute sa scolarité primaire et secondaire en français et vient d'une famille endogame où tous les membres parlent le français à la maison. Il fréquente le Collège Algonquin, établissement à vocation anglaise, parce que son programme n'est pas offert en français dans sa région, en Ontario. Il dit n'avoir aucune occasion de parler français dans la vie quotidienne : «Pis il n'y a rien au Collège Algonquin qui est fait en français. Donc, il n'y a pas personne làbas pour parler en français partout au collège. » Les trois autres participants inscrits dans des universités de langue anglaise ont également confié avoir peu d'interactions, voire aucune, en français.

Outre les problèmes liés à l'apprentissage et à la socialisation en français, certains participants ont également soulevé l'importance d'une reconnaissance de la pluralité des réalités de la francophonie canadienne. En effet, les étudiants franco-ontariens souhaitent que le matériel pédagogique reflète mieux leurs vécus. Constance, qui poursuit ses études à La Cité collégiale, s'exprime ainsi :

Je pense que ma plus grande critique, ça serait par rapport aux enseignants surtout québécois qui ne comprenaient pas notre réalité par exemple pis qui donnaient constamment des exemples de Montréal, ou des exemples québécois. [...] Toute la partie de sensibilisation à la culture franco-ontarienne, je pense que c'est important.

L'étudiante évoque aussi le fait que le français parlé des Franco-Ontariens est parfois décrit comme inadéquat par les francophones issus de situations majoritaires : bien que, comme mentionné plus haut, l'expression de la langue française soit très variée au Canada (Boudreau, 2014; Boudreau \& Dubois, 2001; Chevalier, 2008; Lozon, 2002), certains professeurs semblent ne pas prendre cette diversité en considération en exigeant un français dit «standard ». Elle rapporte par ailleurs que certains professeurs et chargés de cours vont même jusqu'à encourager des étudiants à se tourner vers des programmes 
en anglais plutôt qu'en français. D’autres répondants ont également mentionné ce que Larouche et Hinch (2012) appellent l'intimidation linguistique, dont les incidents génèrent un sentiment d'insécurité linguistique chez plusieurs étudiants francophones en situation minoritaire (LeBlanc, 2010).

Pis il y avait aussi des profs qui, je pense, disent aux étudiants : " va étudier en anglais » à cause de leurs accents. Mais c'est des gens dont la langue maternelle est le français. Ils ont fait leurs études en français au secondaire, mais ils ont un accent qui est par exemple du sud de l'Ontario.

Néanmoins, si de telles expériences négatives au niveau postsecondaire peuvent produire du mécontentement, elles peuvent parfois aussi contribuer à l'éveil de la conscience identitaire des participants. Par exemple, c'est en étant confronté à son statut minoritaire dans une université à vocation bilingue que Thierry dit avoir pris conscience qu'il était Franco-Ontarien.

Mais vraiment ce qui a marqué de façon indubitable le fait que je m'identifie comme Franco-Ontarien, c'était une fois arrivé au postsecondaire. [...] Parce que là, c'était la première fois que j'étais confronté dans un élément où je passais le plus grand temps de la journée minoritaire. C'est-à-dire les autres, l'école élémentaire, secondaire, c'était majoritairement francophone. C'était des institutions francoontariennes. Mais là, le postsecondaire, c'est une institution bilingue qui renforce le sentiment de minorité qui est déjà vécu à l'extérieur des murs [...], fait que là, ça le renforce encore plus.

Tout commeles expériences de socialisation au cours des études primaires et secondaires, les collèges et les universités peuvent encourager les étudiants issus des communautés francophones en situation minoritaire à parler français dans la sphère publique comme dans la sphère privée. Plusieurs des participants souhaiteraient que les établissements postsecondaires créent des espaces où le français serait prédominant et où ils seraient en mesure de débattre des expériences propres aux communautés francophones en situation minoritaire, tout en étant inclusifs à l'égard des diverses variétés de français parlées.

\section{Conclusion}

Les entretiens analysés dans cette étude dévoilent certains aspects moins connus du débat sur le rôle des établissements postsecondaires pour les minorités de langue officielle. La dimension microsociologique del'expérience postsecondaire bilingue des francophones en Ontario met en lumière des problèmes d'accès à des cours en français, de qualité de la langue d'instruction, de possibilités de socialisation en français et d’insécurité linguistique. Les participants inscrits dans des établissements postsecondaires à vocation française se disent satisfaits de leur environnement linguistique, tandis que ceux des établissements postsecondaires à vocation anglaise expriment un sentiment d'isolement linguistique. En effet, bien que les jeunes Franco-Ontariens s’identifient de plus en plus comme bilingues, plusieurs participants ont exprimé le souhait d'apprendre et de socialiser davantage en français. De plus, des participants ont signalé qu'ils souhaitent que leurs professeurs reconnaissent la légitimité des différentes variétés de français parlées au Canada et qu’ils intègrent plus d'exemples propres aux minorités dans le curriculum de leurs cours. 
Certains extraits d'entretiens rapportés témoignent du désir qu'ont plusieurs étudiants franco-ontariens de poursuivre leur éducation postsecondaire en français, mais aussi des difficultés qu'ils rencontrent au moment d'accéder à des cours en français, ce qui confirme les constats du rapport du Commissariat aux services en français de 2012. Phénomène peut-être moins connu, certains participants rapportent également que, parfois, la piètre qualité de la langue d'instruction peut rendre leur apprentissage difficile.

Certaines solutions peuvent être envisagées. Plusieurs participants considèrent par exemple que les établissements à vocation bilingue comme l'Université d'Ottawa devraient accroître les possibilités de socialisation exclusivement en français. De plus, afin de créer un environnement d'apprentissage linguistique inclusif, les professeurs pourraient inclure plus d'exemples franco-ontariens dans le matériel pédagogique enseigné et laisser une plus grande place aux différentes variétés de français parlées au Canada par leurs étudiants. La Fédération de la jeunesse franco-ontarienne (2014) suggère à ce propos la mise sur pied d'un atelier obligatoire incluant des éléments d'histoire, de culture et de défis auxquels sont confrontés les francophones vivant en situation minoritaire pour sensibiliser les professeurs et chargés de cours aux réalités de leurs étudiants. Larouche et Hinch (2012) suggèrent en outre le développement d'une campagne de sensibilisation auprès de l'ensemble des étudiants sur la diversité de la francophonie canadienne pour promouvoir la sécurité linguistique.

Une étude qualitative facilite une compréhension approfondie des processus sociaux qui caractérisent le vécu quotidien des participants. Cependant, les résultats présentés ne peuvent être généralisés à l'ensemble des perspectives des étudiants postsecondaires franco-ontariens. Pour dresser un portrait global, il serait nécessaire d'effectuer une enquête sous forme de sondage dans l'ensemble des établissements postsecondaires en Ontario. Plusieurs francophones fréquentent des établissements de langue anglaise, et les besoins spécifiques qu'ils rencontrent lors de leur transition de l'école secondaire de langue française aux études postsecondaires en anglais sont encore peu connus. Une attention toute particulière pourrait aussi être portée aux perspectives de l'ensemble des étudiants inscrits dans des établissements offrant des programmes en français : étudiants internationaux, francophiles en immersion française et membres des communautés francophones en situation minoritaire. Une telle enquête permettrait d'effectuer un tour d'horizon des réussites et des besoins des établissements postsecondaires et des communautés environnantes. À l'heure de la mondialisation, les conclusions de cette étude concernant l'insécurité linguistique et la socialisation sont pertinentes pour les autres communautés francophones hors Québec, les minorités linguistiques nationales ainsi que les minorités culturelles préoccupées par la rétention et la transmission d'un patrimoine culturel spécifique.

\section{Note}

1. Cette étude a été menée par l'auteure uniquement et a été financée par le Conseil de recherches en sciences humaines (CRSH), les Bourses d'études supérieures de l'Ontario (BESO) et l'Institut canadien de recherche sur les minorités linguistiques (ICRML). 


\section{Remerciements}

Je tiens à remercier $\mathrm{D}^{\mathrm{r}}$ Scott Davies, $\mathrm{D}^{\mathrm{r}}$ Jeffrey Denis et Jean-François Nault pour leurs suggestions constructives. J'aimerais également remercier $\mathrm{D}^{\mathrm{r}}$ Normand Labrie de m'avoir invitée à présenter une version antérieure de cet article lors de la table ronde et du lancement du livre L'accès des francophones aux études postsecondaires en Ontario : perspectives étudiantes et institutionnelles au Centre de recherche en éducation franco-ontarienne. Finalement, j'aimerais remercier chaleureusement l'ensemble des participants de ce projet de recherche.

\section{Références}

Alexander, J. C. (2013). Struggling over the mode of incorporation: Backlash against multiculturalism in Europe. Ethnic and Racial Studies, 36(4), 531-556.

Anderson, E. (1999). Code of the street: Decency, violence, and the moral life of the inner city. New York, NY : W. W. Norton and Company.

Anderson, L. \& Snow, D.A. (2001). L'exclusion sociale et le soi : une perspective d'interactionnisme symbolique. Sociologie et sociétés, 33(2), 13-27.

Blumer, H. (1969). Symbolic interactionism: Perspective and method. New Jersey, NY : Prentice Hall.

Boudreau, A. (2014). Des voix qui se répondent : analyse discursive et historique des idéologies linguistiques en Acadie : l'exemple de Moncton. Minorités linguistiques et société/Linguistic Minorities and Society, 4, 175-199.

Boudreau, A. \& Dubois, L. (2001). Langues minoritaires et espaces publics : le cas de l'Acadie du Nouveau-Brunswick. Estudios de Sociolingüística, 2(1), 37-60.

Bourhis, R.Y., Sioufi, R., \& Sachdev, I. (2012). Ethnolinguistic interaction and multilingual communication. Dans H. Giles (dir.), The Handbook of Intergroup Communication (p. 100115). New York, NY : Routledge.

Breton, R. (1964). Institutional completeness of ethnic communities and personal relations of immigrants. American Journal of Sociology, 7O(2), 193-205.

Castonguay, C. (2002). Note critique : pensée magique et minorités francophones. Recherches sociographiques, 43(2), 369-380.

Chevalier, G. (2008). Les Français du Canada : faits linguistiques, faits de langue. Alternative francophone, 1(1), 80-97.

Churchill, S. (2016). Progrès notables et persistance de problèmes structuraux : le postsecondaire français en Ontario. Dans N. Labrie \& S.A. Lamoureux (dir.), L'accès des francophones aux études postsecondaires en Ontario : perspectives étudiantes et institutionnelles (p. 13-26). Sudbury, Canada : Éditions Prise de Parole.

Commissariat aux services en français. (2012). Rapport d'enquête - L'état de l'éducation postsecondaire en langue française dans le Centre-Sud-Ouest de l'Ontario : pas d'avenir sans accès. Toronto, Canada : Gouvernement de l'Ontario.

Corbeil, J.-P., Chavez, B., \& Pereira, D. (2010). Portrait des minorités de langue officielle au Canada : les anglophones du Québec. Ottawa, Canada : Statistique Canada. 
Dallaire, C. (2008). La stabilité des discours identitaires et la représentation de la culture dansla reproduction del'appartenance francophone chezlesjeunes. Francophonies d'Amérique, 26, 357-381.

Dallaire, C. \& Denis, C. (2005). Asymmetrical hybridities: Youths at francophone games in Canada. Cahiers canadiens de sociologie/Canadian Journal of Sociology, 3O(2), 143-168.

Dwyer, S. C. (2000). Overcoming obstacles to education: The experience of women university students diagnosed with attention-deficit/hyperactivity disorder. Revue canadienne d'enseignement supérieur/Canadian Journal of Higher Education, 3o(1), 123-148.

Fédération de la jeunesse franco-ontarienne. (2014). Pour s'exprimer dans notre langue : consultation sur l'insécurité linguistique et la jeunesse franco-ontarienne. Ottawa, Canada : Fédération de la jeunesse franco-ontarienne.

Frenette, N. \&t Quazi, S. (1996). Accessibilité et participation des francophones de l'Ontario à l'éducation postsecondaire, 1979-1994. Sudbury, Canada : Collège Boréal.

Gérin-Lajoie, D. (2011). Belonging to two worlds at once. Dans J. Jedwab et R. Landry (dir.), Life after forty: Official languages policy in Canada (p. 179-198). Montréal, Canada : McGill-Queen's University Press.

Gérin-Lajoie, D. (2014). Identité et sentiment d'appartenance chez les jeunes anglophones de Montréal. Recherches sociographiques, 55(3), 467-484.

Giles, H., Bourhis, R.Y., \& Taylor, D. M. (1977). Towards a theory of language in ethnic group relations. Dans H. Giles (dir.), Language ethnicity and intergroup relations (p. 307-348). Londres, R.-U. : Academic Press.

Gouvernement de l'Ontario. (2014). L'entente Canada-Ontario relative à l'enseignement dans la langue de la minorité et à l'enseignement de la langue seconde officielle 2013-2014 à 2017-2018. Récupéré de http://www.edu.gov.on.ca/fre/ amenagement/ententeAgreementEdFr.pdf

Hall, P.A. \& Lamont, M. (2013). Why social relations matter for politics and successful societies. The Annual Review of Political Science, 16(1), 49-71.

Hampton, M. \& Roy, J. (2002). Strategies for facilitating success of First Nations students. Revue canadienne d'enseignement supérieur/Canadian Journal of Higher Education, 32(3), 1-28.

Kilbride, K. M. \& D’Arcangelo, L. (2002). Meeting immigrant community college students' needs on one Greater Toronto area college campus. Revue canadienne d'enseignement supérieur/Canadian Journal of Higher Education, 32(2), 1-26.

Labrie, N. \& Lamoureux, S. A. (2016). L'accès des francophones aux études postsecondaires en Ontario : perspectives étudiantes et institutionnelles. Sudbury, Canada : Éditions Prises de Parole.

Labrie, N., Lamoureux, S., \& Wilson, D. (2009). L'accès des francophones aux études postsecondaires en Ontario : le choix des jeunes. Toronto, Canada : Centre de recherches en éducation franco-ontarienne. 
Landry, R. (2014). La vie dans une langue officielle minoritaire au Canada. Québec, Canada : Presses de l'Université Laval.

Landry, R., Deveau, K., \& Allard, R. (2008). Dominance identitaire bilingue chez les jeunes francophones en situation minoritaire. Revue canadienne de recherche sociale/ Canadian Journal for Social Research, 1, 2-10.

Larouche, F. \& Hinch, C. (2012). Bâtir sur le roc : prévenir l'insécurité linguistique. Proposition d'atelier sur l'insécurité linguistique dans le cadre du Congrès d'ACELF.

LeBlanc, M. (2010). Le français, langue minoritaire, en milieu de travail : des représentations linguistiques à l'insécurité linguistique. Nouvelles perspectives en sciences sociales : Revue internationale de systémique complexe et d'études relationnelles, 6(1), 17-63.

Lozon, R. (2002). Représentations et sentiments linguistiques des francophones du Sud-Ouest de l'Ontario et la reproduction des variétés de français. Francophonies d'Amérique, 14, 55-70.

Mead, G. H. (1934). Mind, self, and society. (Édité par Charles W. Morris). Chicago, IL : University of Chicago Press.

Ministère de l'Enseignement supérieur et de la Formation professionnelle (2011). Politique d'aménagement linguistique: a policy framework for French-language postsecondary education and training in Ontario. Toronto, Canada : Gouvernement de l'Ontario.

Office des affaires francophones. (2009). Redéfinition de la population francophone. Toronto, Canada : Gouvernement de l'Ontario.

Office des affaires francophones. (2012). Portrait de la communauté francophone de l'Ontario. Toronto, Canada : Gouvernement de l'Ontario.

Pidgeon, M., Archibald, J.-A., \& Hawkey, C. (2014). Relationships matter: Supporting Aboriginal graduate students in British Columbia, Canada. Revue canadienne d'enseignement supérieur/Canadian Journal of Higher Education, 44(1), 1- 21.

Pilote, A. \& Joncas, J.-A. (2016). La construction identitaire linguistique et culturelle durant un programme universitaire d'éducation en français en milieu minoritaire : le cas de cinq étudiants fransaskois. Minorités linguistiques et société/ Linguistic Minorities and Society, 7, 142-169.

Pilote, A. \& Magnan, M.-O. (2012). La construction identitaire des jeunes francophones en situation minoritaire au Canada : négociation des frontières linguistiques au fil du parcours universitaire et de la mobilité géographique. Cahiers canadiens de sociologie/Canadian Journal of Sociology, 37(2), 169-195.

Pilote, A. \& Magnan, M.-O. (2014). La fréquentation universitaire : comparaison entre les minorités de langue officielle au Canada. Dans R. Landry (dir.), La vie dans une langue officielle minoritaire au Canada (p. 147-172). Québec, Canada : Presses de l'Université Laval. 
Pilote, A., Magnan, M.-O., \& Vieux-Fort, K. (2010). Identité linguistique et poids des langues : une étude comparative entre des jeunes de milieu scolaire francophone au Nouveau-Brunswick et anglophone du Québec. Nouvelles perspectives en sciences sociales : Revue internationale de systémique complexe et d'études relationnelles, 6(10), 65-98.

Rae, Hon. B.(2005). L'Ontario : chefdefileenéducation, rapportetrecommandations. Toronto, Canada : Gouvernement de l'Ontario.

Small, M. L. (2009). 'How many cases do I need?': On science and the logic of case selection in field-based research. Ethnography, 1O(1), 5-38.

Van Rhijn, T.M., Quosai, T.S., \& Lero, D.S. (2011). A profile of undergraduate student parents in Canada. Revue canadienne d'enseignement supérieur/Canadian Journal of Higher Education, 41(3), 59-80.

Weber, M. (1949). The Methodology of the Social Sciences. Glencoe, IL : Free Press.

\section{Biographie}

Johanne Jean-Pierre

School of Child and Youth Care

Université Ryerson

jjeanpierre@ryerson.ca

Johanne Jean-Pierre, détentrice d'un doctorat en sociologie de l'Université McMaster, est professeure adjointe à l'École d'éducation spécialisée (Child and Youth Care) de l'Université Ryerson. Ses principaux intérêts de recherche portent sur les inégalités, l'éducation et le vécu culturel des enfants et des jeunes issus des minorités de langue officielle, des communautés afro-canadiennes et de l'immigration. 